\title{
Nationwide "Hospital Emergent Capability Accreditation by Level-Stroke" Improves Stroke Treatment in Taiwan
}

\author{
Tain-Junn Cheng, ${ }^{\text {a,b }}$ Giia-Sheun Peng, ${ }^{c}$ Wei-Siang Jhao, ${ }^{d}$ Jiunn-Tay Lee, ${ }^{e}$ Tsung-Hsi Wang ${ }^{f}$
}

\begin{abstract}
aDepartments of Neurology and Occupational Medicine, Chi Mei Medical Center, Tainan, Taiwan, Republic of China
${ }^{b}$ Department of Hospital and Health Care Administration, College of Recreation and Health Management, Chia Nan University of Pharmacy and Science, Tainan, Taiwan, Republic of China

'Division of Neurology, Department of Internal Medicine, Taipei Veterans General Hospital, Hsinchu Branch, Hsinchu County, Taiwan, Republic of China

${ }^{d}$ Department of Medical Affairs, Ministry of Health and Welfare, Executive Yuan, Republic of China, Taipei, Taiwan, Republic of China 'Department of Neurology, Tri-Service General Hospital, National Defense Medical Center, Taipei, Taiwan, Republic of China

${ }^{f}$ Ministry of Health and Welfare, Executive Yuan, Republic of China, Taipei, Taiwan, Republic of China
\end{abstract}

Background and Purpose Recombinant tissue plasminogen activator (rtPA) is one of the proven therapies that improve the outcome of patients with acute ischemic stroke (AIS). In 2009, the Ministry of Health and Welfare, Executive Yuan, Republic of China, launched the project "Hospital Emergent Capability Accreditation by Level-Stroke (HECAL-Stroke)" to improve AIS treatment in Taiwan. The current study was performed to determine whether the project launched by the government was effective in promoting rtPA therapy among AIS patients.

Methods All participating hospitals were verified and designated as "heavy duty (HD)," "moderate duty (MoD)," or "medium duty (MeD)" according to the stroke center criteria. Four annual indices (rates of treatment, protocol adherence, in-time treatment, and complications) were recorded from 2009 to 2014 as outcome measures. The data were analyzed using the $\chi^{2}$ test for significance.

Results The number of certified hospitals progressively increased from 74 to 112 during the 6-year period and finally consisted of $33 \mathrm{HD}, 9 \mathrm{MoD}$ and $70 \mathrm{MeD}$ hospitals in 2014. The annual intravenous rtPA treatment rate increased significantly from 3.0\% in 2009 to $4.5 \%$ in 2014. The protocol adherence rates were $95.7 \%$ in the $\mathrm{HD}$ group, $92.4 \%$ in the MoD group and $72.8 \%$ in the MeD group. The annual in-time treatment rate significantly improved from $26.0 \%$ in 2009 to $60.1 \%$ in 2014 . The overall symptomatic intracranial hemorrhagic rate after rtPA treatment was $8.6 \%$.

Conclusions Initiation of the HECAL-Stroke project by the government significantly improved rtPA treatment in Taiwan.

Keywords Ischemic stroke; Thrombolysis; Tissue-type plasminogen activator; Hospital Emergent Capability Accreditation by Level-Stroke; Taiwan
Correspondence: Tsung-Hsi Wang Ministry of Health and Welfare, Executive Yuan, Republic of China, 10F, No. 488, Sec. 6, Zhongxiao E. Rd., Nangang Dist., Taipei City, 11558, Taiwan, Republic of China

Tel: +886-2-85907750

Fax: +886-2-8590-7013

E-mail: Joyce12wang@gmail.com

Received: October 27, 2016

Revised: March 30, 2017

Accepted: April 23, 2017

This work was partially supported by The Ministry of Health and Welfare, Executive Yuan, Republic of China, and the Taiwan Neurological Society and by Taipei Veterans General Hospital, Hsinchu Branch (VHCTRD-2016-8 and VHCT-RD-2017-6).

The authors have no conflicts of interest. 


\section{Introduction}

Stroke has been one of the top three causes of death and the most common cause of disability among adults in Taiwan over the past 30 years. ${ }^{12}$ The impact of the stroke-related medical and economic burdens on families and society is considerable. Therefore, the promotion of public awareness for the prevention of stroke and the optimization of treatments for patients with acute ischemic stroke (AIS) to minimize the overall burden of stroke-related dependency have become important healthcare missions of the Ministry of Health and Welfare (MOHW), Executive Yuan, Republic of China.

Intravenous recombinant tissue plasminogen activator (IV rtPA) therapy within a limited time window ( 3 to 4.5 hours after stroke symptom onset) is the most important treatment with proven clinical efficacy and provides cost savings for select patients with AIS., ${ }^{3,4}$ Thrombolytic therapy with rtPA was approved for AIS in 2002 by the Taiwan Food and Drug Administration, and its efficacy has been thoroughly evaluated to improve the quality of stroke care in Taiwan. ${ }^{5}$ The Taiwan Stroke Registration program conducted by the Taiwan Stroke Society from 2006 to 2008 was the first nationwide effort in Taiwan to establish a reliable national stroke database to assess the quality of stroke care and to identify areas that required improvement. The study demonstrated that only $1.5 \%$ of patients with ischemic stroke and $8.8 \%$ of patients arriving at the hospital within 2 hours of stroke onset received IV rtPA treatment. The proportion of patients who received rtPA therapy in the Taiwan Stroke Registration was significantly lower than the proportion in the USA according to the Get With the Guidelines-Stroke study. ${ }^{6}$ However, increasing evidence has indicated that the establishment of primary stroke centers (PSCs) and certification by professionals is effective in increasing the use of rtPA therapy, which is regarded as an important measure of the quality of stroke care.? Therefore, the nationwide project "Hospital Emergent Capability Accreditation by Level-Stroke (HECAL-Stroke)" launched by the MOHW in Taiwan in 2009 encouraged hospitals to establish a PSC and to set a standard requirement for PSC accreditation. ${ }^{8}$ The current study was to evaluate the impact of the nationwide HECAL-Stroke project on the improvement of IV rtPA treatment in Taiwan.

\section{Methods}

\section{Hospital enrollment}

HECAL is a nationwide hospital accreditation project launched by the MOHW in 2009 in Taiwan. ${ }^{8}$ The program evaluates six domains of hospital accreditation, including medical manage- ment in the emergency department, stroke, acute coronary syndrome, major trauma, gynecological and pediatric emergency, and intensive care unit. The evaluation process was conducted by an on-site peer reviewing committee according to the criteria of each domain from a nationwide consensus. The adequacies of the emergency department and intensive care unit facilities are basic requirements for participation in the nationwide hospital accreditation project. Initially, all participating hospitals were certified as "heavy duty (HD)" when they met all of the requirements for the acute management of stroke, acute coronary syndrome, major trauma, and gynecological and pediatric emergency and as "medium duty (MeD)" when they met only the requirement for the management of one of these conditions according to the audit results of the on-site peer review (initially and then every 4 years). In 2011, the additional certification "moderate duty (MoD)" was introduced to define a hospital meeting the requirements for the management of more than one of the conditions. In the current study, we enrolled all participating hospitals that met the HECAL criteria for acute stroke management in the HECALStroke project to evaluate outcome measurements from 2009 to 2014 .

\section{Data collection}

The performance of rtPA treatment was regarded as the major outcome measure for the HECAL-Stroke project. The participating and certified hospitals were requested to report annual data to the MOHW, including all six items listed below.

1. Number of patients with AIS

2. Number of patients with AIS arriving at the hospital within 3 hours who were eligible for IV rtPA treatment (Supplementary Methods 1)

3. Number of patients with AIS receiving IV rtPA treatment

4. Number of patients with AIS who were eligible for rtPA therapy and received the treatment

5. Number of patients with AIS who received IV rtPA treatment within 60 minutes of arrival at the emergency department

6. Number of symptomatic intracranial hemorrhage $(\mathrm{SICH})$ events after rtPA therapy (SICH was defined as an intracranial hemorrhage detected on follow-up imaging and no less than 2 scales of deterioration according to the National Institutes of Health Stroke Scale within 36 hours after rtPA treatment) ${ }^{4}$

We assessed the following measures (A-D) as quality indicators:

A. Treatment rate, indicating the proportion of patients with AIS receiving rtPA therapy (calculated as the number of 


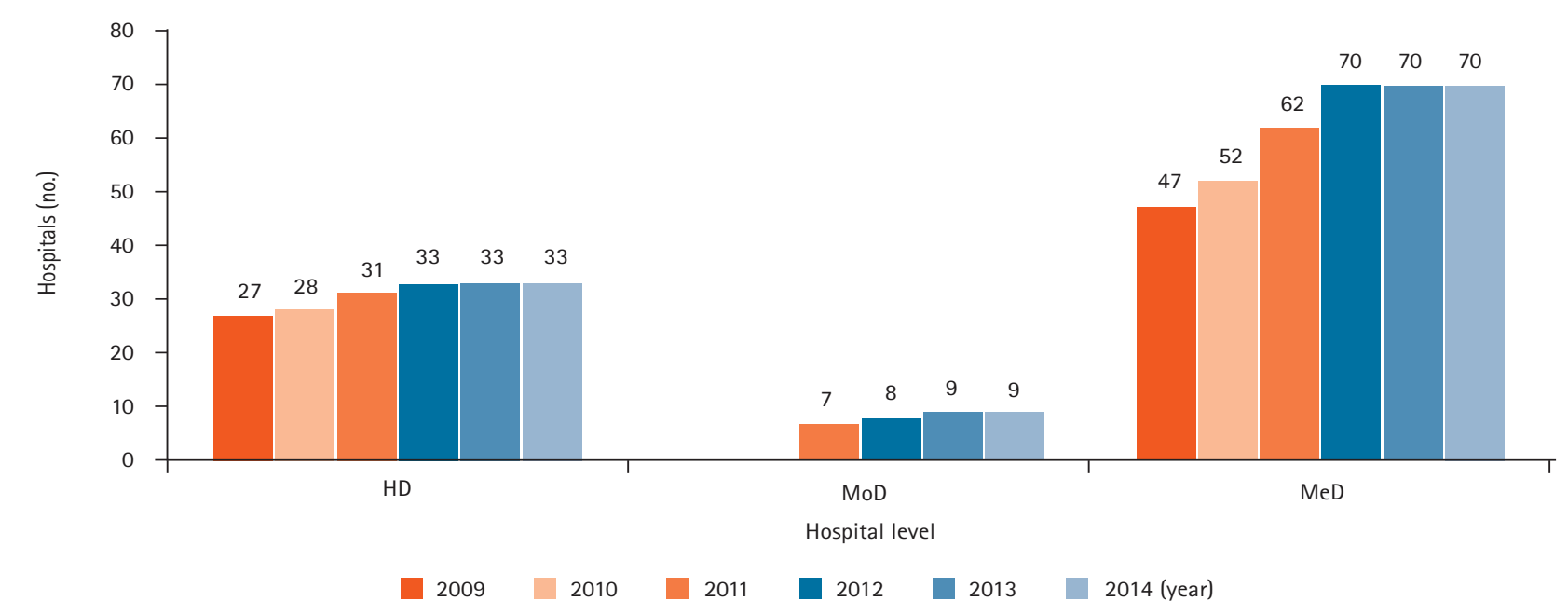

Figure 1. Participating hospitals in the different levels from 2009 to 2014. HD, heavy duty; MeD, medium duty; MoD, moderate duty.

item 3 divided by the number of item 1 and then multiplied by $100 \%)$

B. Protocol adherence rate, indicating the proportion of patients with AIS who qualified for rtPA therapy and received the treatment (calculated as the number of item 4 divided by the number of item 2 and then multiplied by 100\%)

C. In-time treatment rate, indicating the proportion of patients with AIS who received IV rtPA treatment within 60 minutes (calculated as the number of item 5 divided by the number of item 3 and then multiplied by 100\%)

D. SICH rate, indicating the proportion of patients with $\mathrm{SICH}$ after IV rtPA therapy (calculated as the number of item 6 divided by the number of item 3 and then multiplied by 100\%)

To achieve HECAL-Stroke accreditation, all hospitals must reach the minimal target goals within a four-year period, including a $1 \%$ treatment rate, $50 \%$ protocol adherence rate, and $30 \%$ in-time treatment rate every year. ${ }^{8}$

The study protocols were approved by the Institutional Review Board of Chi Mei Medical Center (approval No.: 10410E07) for data analysis without personal patient identifiers.

\section{Statistical analysis}

All categorical variables were expressed as numbers and percentages, and the indices were presented as percentages. Pearson's $\chi^{2}$ test or Fisher's exact test was used to analyze differences among two or three groups. All statistical tests were performed at the two-tailed significance level of 0.05. All data processing and statistical analyses were performed with the EXCEL ${ }^{\oplus}$ statistical software (Microsoft ${ }^{ø}$ Office 2010, Microsoft
Corporation, Seattle, WA, USA).

\section{Results}

The number of certified hospitals increased from 74 in 2009 to 112 in 2014 and included 33 HD hospitals, 9 MoD hospitals and $70 \mathrm{MeD}$ hospitals in 2013 and 2014 (Figure 1). Nearly all of the hospitals with the facilities to manage AIS patients in Taiwan were enrolled. Therefore, the study could reflect the findings of the nationwide project to improve acute stroke management for patients with AIS during the period from 2009 to 2014.

An estimated 31,000 patients experienced AIS yearly in Taiwan (Table 1). The four quality indicators were collected annually from the $H D, M o D$, and MeD summation data from 2009 to 2014 (Supplementary Table 1, Supplementary Figure 1). The annual IV rtPA treatment rate significantly increased from $3.0 \%$ in 2009 to $4.2 \%$ in 2011 and then gradually increased to 4.5\% in 2014 (Table 1, Figure 2A). A comparison of the treatment rates among the three hospital levels showed that the highest rate was $5.7 \%$ in the MoD group, which was significantly higher compared to the other two groups (Table 2, Figure 3A).

The overall protocol adherence rate was $87.1 \%$ (Table 1). The protocol adherence rates were $95.7 \%$ at the HD level, $92.4 \%$ at the MoD level and $72.8 \%$ at the MeD level, which represented significant differences (Table 2, Figure 3B). The annual protocol adherence rate was significantly lower in $2011(83.8 \%)$ than in 2010 (89.8\%) and 2012 (86.8\%) (Table 1 , Figure $2 \mathrm{~B}$ ), which might be attributed to the poor protocol adherence, such as rtPA prescribed in patients older 
Table 1. Annual data and indicators for AIS patients receiving rtPA therapy from 2009 to 2014 in the project "Hospital Emergent Capability Accreditation by Level-Stroke"

\begin{tabular}{|c|c|c|c|c|c|c|c|c|}
\hline & 2009 & 2010 & 2011 & 2012 & 2013 & 2014 & Total & Overall \\
\hline Hospital no. & 74 & 80 & 100 & 111 & 112 & 112 & NA & \\
\hline \multicolumn{9}{|l|}{ Items } \\
\hline 1. AIS & 12,481 & 16,905 & 26,707 & 31,558 & 31,499 & 30,771 & 149,921 & \\
\hline 2. Eligible for rtPA & 360 & 591 & 1,247 & 1,436 & 1,431 & 1,461 & 6,526 & \\
\hline 3. rtPA administration & 377 & 640 & 1,121 & 1,350 & 1,328 & 1,370 & 6,186 & \\
\hline 4. Qualifying for rtPA criteria & 319 & 531 & 1,045 & 1,247 & 1,262 & 1,278 & 5,682 & \\
\hline 5. Administration of rtPA within 60 min. & 98 & 242 & 559 & 747 & 778 & 823 & 3,247 & \\
\hline 6. SICH & 28 & 62 & 86 & 122 & 107 & 124 & 529 & \\
\hline \multicolumn{9}{|l|}{ Indicator (item no./item no.) (\%) } \\
\hline Treatment rate (3/1) & $3.0^{\circ}$ & $3.8^{*}+$ & $4.2^{+}$ & 4.3 & 4.2 & 4.5 & & 4.1 \\
\hline Protocol adherence rate (4/2) & 88.6 & $89.8^{+}$ & $83.8^{+, *}$ & $86.8^{\S}$ & 88.2 & 87.5 & & 87.1 \\
\hline In-time treatment rate (5/3) & $26.0^{\circ}$ & $37.8^{*+}$ & $49.9^{t, *}$ & $55.3^{\S}$ & 58.6 & 60.1 & & 52.5 \\
\hline $\mathrm{SICH}$ rate $(6 / 3)$ & 7.4 & 9.7 & 7.7 & 9.0 & 8.1 & 9.1 & & 8.6 \\
\hline
\end{tabular}

AIS, acute ischemic stroke; rtPA, recombinant tissue plasminogen activator; no., number; NA, not applicable; min., minutes; SICH, symptomatic intracranial hemorrhage.

${ }^{*} P<0.05,2009$ vs. $2010 ;{ }^{+} P<0.05,2010$ vs. $2011 ;{ }^{\ddagger} P<0.05,2011$ vs. 2012. Significant differences between the two groups of the index were analyzed with a $\chi^{2}$ test with $P<0.05$.
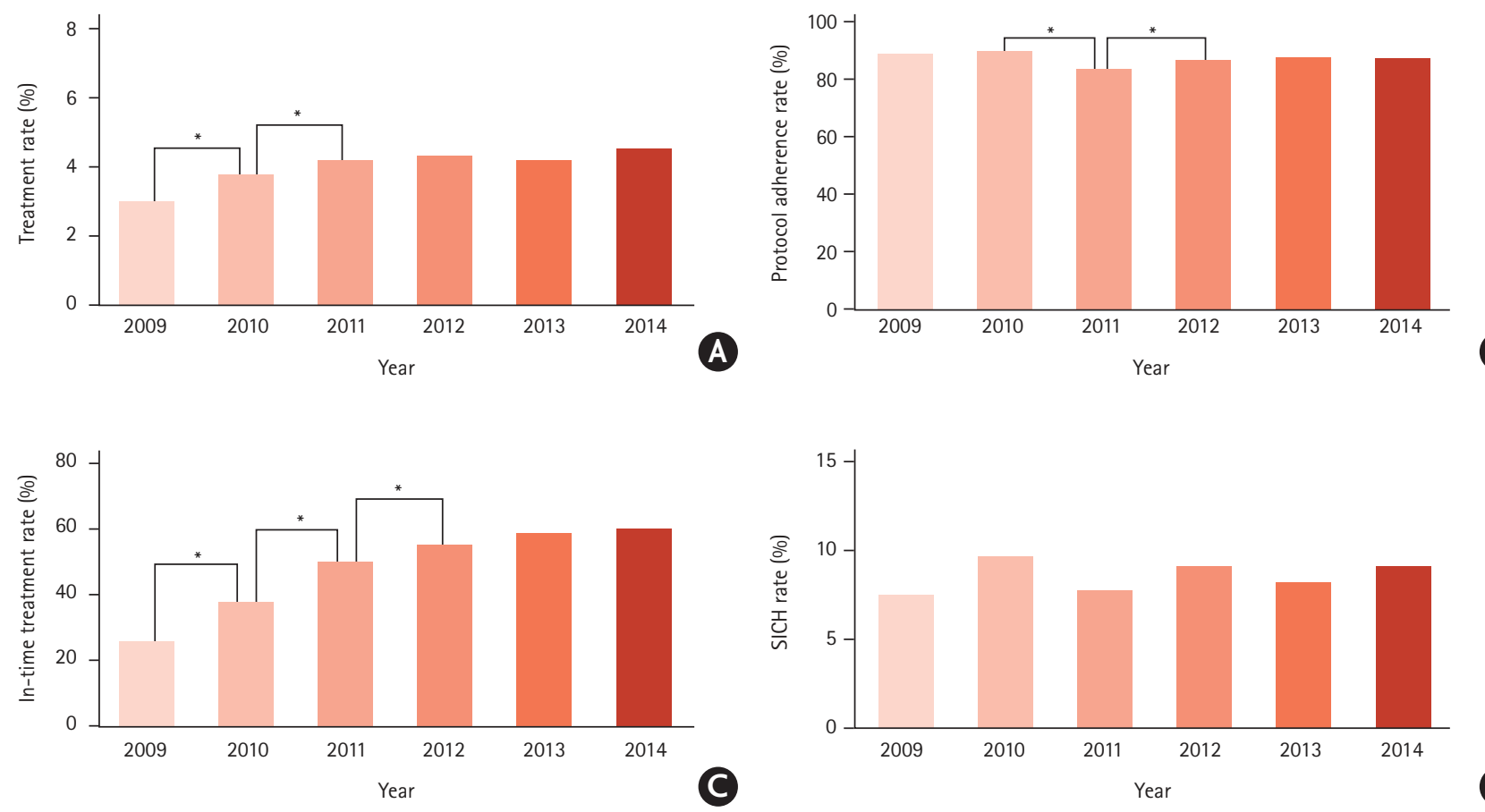

Figure 2. Annual indicators for AIS patients receiving rtPA therapy from 2009 to 2014 in the project of "Hospital Emergent Capability Accreditation by LevelStroke." (A) Treatment rate, (B) protocol adherence rate, (C) in-time treatment rate, and (D) SICH rate. AIS, acute ischemic stroke; rtPA, recombinant tissue plasminogen activator; $\mathrm{SICH}$, symptomatic intracranial hemorrhage. ${ }^{*}$ Represents a significant difference between groups analyzed with a $\chi^{2}$ test with $P<0.05$.

than 80 years old or 3-4.5 hours of AIS onset, in the MeD group (Table 2, Figure 3B, Supplementary Table 1, Supplementary Figure 1B).

The annual in-time rates significantly improved from $26.0 \%$ in 2009 (24.5\% in the HD group and $34.5 \%$ in the MeD group) to $55.3 \%$ in 2012 and then gradually improved to $60.1 \%$ (57.5\% in the HD group, $61.2 \%$ in the MoD group and 63.6\% in the MeD group) in 2014 (Table 1, Figure 2C, Supplementary 
Table 2. Data and indicators for AIS patients receiving rtPA therapy at different hospital levels from 2009 to 2014 in the project of "Hospital Emergent Capability Accreditation by Level-Stroke"

\begin{tabular}{|c|c|c|c|c|c|}
\hline & $\mathrm{HD}$ & MoD & MeD & Total & Overall \\
\hline \multicolumn{6}{|l|}{ Items } \\
\hline 1. AIS & $94,354(62.9)$ & $7,479(5.0)$ & $48,088(32.1)$ & 149,921 & \\
\hline 2. Eligible for rtPA & $3,729(57.1)$ & $396(6.1)$ & $2,401(36.8)$ & 6,526 & \\
\hline 3. rtPA administration & $3,973(64.2)$ & $430(7.0)$ & $1,783(28.8)$ & 6,186 & \\
\hline 4. Qualifying for rtPA criteria & $3,567(62.8)$ & $366(6.4)$ & $1,749(30.8)$ & 5,682 & \\
\hline 5. Administration of rtPA within $60 \mathrm{~min}$. & $2,033(62.6)$ & $241(7.4)$ & $973(30.0)$ & 3,247 & \\
\hline 6. $\mathrm{SICH}$ & $229(43.3)$ & $22(4.2)$ & $278(52.6)$ & 529 & \\
\hline \multicolumn{6}{|l|}{ Indicator (item no./item no.) (\%) } \\
\hline Treatment rate $(3 / 1)^{*}$ & $4.2^{+, *}$ & $5.7^{+, \S}$ & $3.7^{\ddagger, s}$ & & 4.1 \\
\hline Protocol adherence rate $(4 / 2)^{+}$ & $95.7^{\neq, \S}$ & $92.4^{\neq, \|}$ & $72.8^{\S, \|}$ & & 87.1 \\
\hline In-time treatment rate $(5 / 3)^{+}$ & $51.2^{\S}$ & 56.0 & $54.6^{\S}$ & & 52.5 \\
\hline $\mathrm{SICH}$ rate $(6 / 3)^{+}$ & $5.8^{\S}$ & $5.1^{11}$ & $15.6^{\text {s,l1 }}$ & & 8.6 \\
\hline
\end{tabular}

Values are presented as $n(\%)$ unless otherwise indicated.

AIS, acute ischemic stroke; HD, heavy duty; MeD medium duty; MoD, moderate duty; min., minutes; rtPA, recombinant tissue plasminogen activator; SICH, symptomatic intracranial hemorrhage.

${ }^{*}$ Significant differences between two groups of the index analyzed with a $\chi^{2}$ test with $P<0.05 ;{ }^{+} P<0.05 \mathrm{HD}$ vs. MeD; ${ }^{{ }^{P}} P<0.05 \mathrm{HD}$ vs. MoD; ${ }^{{ }^{5}} P<0.05 \mathrm{HD}$ vs. MeD; "P<0.05 MoD vs. MeD.
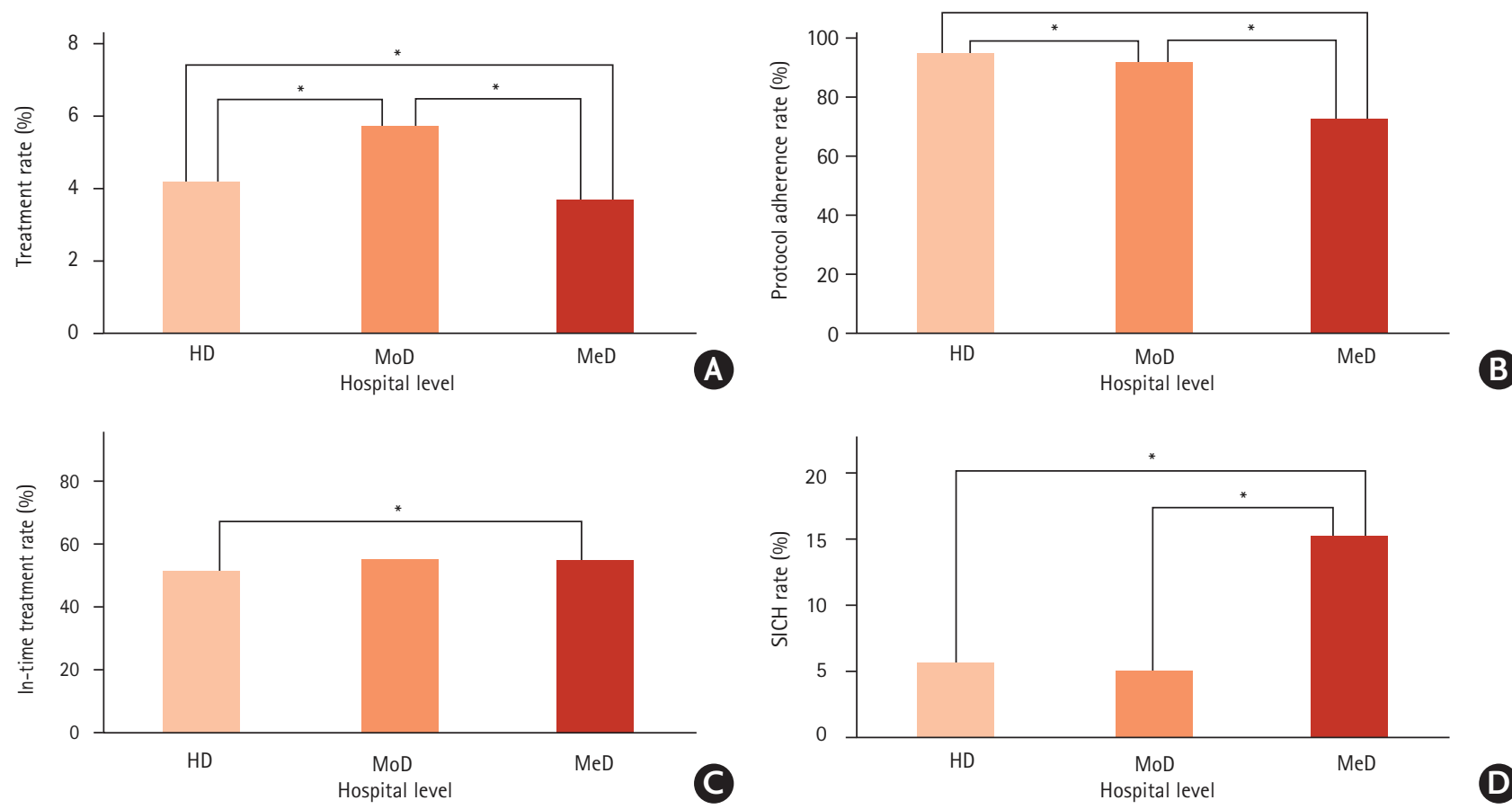

(D)

Figure 3. Indicators for AIS patients receiving rtPA therapy at different hospital levels from 2009 to 2014 in the project of "Hospital Emergent Capability Accreditation by Level-Stroke". (A) Treatment rate, (B) protocol adherence rate, (C) in-time treatment rate, and (D) SICH rate. AIS, ischemic stroke; HD, heavy duty; MeD medium duty; MoD, moderate duty; rtPA, recombinant tissue plasminogen activator; $\mathrm{SICH}$, symptomatic intracranial hemorrhage. ${ }^{*}$ Represents a significant difference between groups analyzed with a $\chi^{2}$ test with $P<0.05$.

Table 1, Supplementary Figure 1C). The overall in-time treatment rates were $51.2 \%$ at the $\mathrm{HD}$ level and $54.6 \%$ at the MeD level, which represented significant difference (Table 2, Figure 3C).
The overall complication rate after IV rtPA treatment was $8.6 \%$, and no significant differences were detected between the years (Table 1, Figure 2D). However, the differences in the 
complication rates were significant, with the highest rate of $15.6 \%$ observed in the MeD group, followed by $5.8 \%$ in the HD group and 5.1\% in the MoD group (Table 2, Figure 3D).

\section{Discussion}

A previous study demonstrated that only $1.5 \%$ of patients with AIS received IV rtPA treatment between 2006 and 2008 in Taiwan. ${ }^{5}$ This study demonstrated that the HECAL-Stroke project launched by the MOHW of Taiwan significantly increased the rtPA treatment rate in patients with AIS from 3.0\% in 2009 to 4.5\% in 2014. Accordingly, the in-time rate of rtPA treatment also significantly increased in all groups (HD, MoD and MeD) from 2009 to 2014. The overall complication rate was 8.6\% in the rtPA-treated patients at all hospital levels, but no significant differences were detected between 2009 and 2014.

Increasing evidence has indicated that the implementation and certification of a PSC may improve the clinical performance of rtPA treatment in AIS patients. ${ }^{910}$ Prior to PSC certification, Kleindorfer et al. found that the rtPA treatment rate was extremely low (approximately 1.8-2.1\% of AIS patients) in both the Medicare Provider Analysis and Review database and the Premier Hospital database between 2001 and 2004. ${ }^{11}$ The treatment rate was significantly higher in PSC-certified hospitals by the Joint Commission on Accreditation of Healthcare Organizations than in uncertified hospitals (5.0\% versus $1.4 \%$ ) in 2010. ${ }^{12}$ Interestingly, Lattimore et al. reported that the establishment of a PSC at a community hospital resulted in a substantial increase in the proportion of AIS patients receiving rtPA therapy, from 1.5\% before certification to $10.5 \% 2$ years after certification. ${ }^{7}$

Earlier evidences inspired the MOHW of Taiwan for launching the project of HECAL-Stoke. The current study indicated that the treatment rate significantly increased not only in the MeD group, which primarily consists of community hospitals, but also in the HD group with academic medical centers. This finding suggests that Taiwan's HECAL-Stroke project for the certification of PSCs was effective in increasing the rtPA treatment rate for AIS patients in both community hospitals and academic medical centers. In addition to increasing the rtPA treatment rate for AIS patients, Taiwan's HECAL-Stroke project significantly increased the in-time treatment rate from 26.0\% in 2009 to $60.1 \%$ in 2014 (Table 1) compared with the in-time treatment rate of 8.8\% from 2006 to 2008 in Taiwan. ${ }^{5}$ This result may indicate that the process of AIS evaluation and treatment changed at many levels after the initiation of Taiwan's HECAL-Stroke project for stroke certification, which facilitated community hospitals and academic medical centers to stan- dardize numerous aspects of stroke care. It could be the main reason for increasing rtPA treatment rate.

The hospitals that did not meet the criteria for stroke centers were not recruited for treatment quality data in the study. Although all hospitals met the criteria for acute stroke management, there were the disparities of treatment outcomes among the stroke centers. The differences of treatment outcomes may be related to patients' demographics and the various levels of hospital designation for stroke care, which includes human and structural resources and integrated pathways for management of acute stroke patients. The MoD hospitals had the significantly highest treatment rate than the $\mathrm{HD}$ and MeD hospitals, but had no higher in-time treatment and protocol adherence rates than the HD hospitals. The reason may be ascribed to the fact that the MoD hospitals are regional and convenient for stroke patients with reduction of prehospital delay. Further exploring the contributors for the disparities of treatment outcomes among various levels of stroke center is needed in the future.

Based on previous evidence, a shorter onset-to-treatment time leads to a better prognosis in AIS patients. ${ }^{13}$ Lindsberg et al. streamlined the triage process in the emergency room (ER) to reduce the onset-to-treatment time and thereby reduced in-hospital delays and enhanced thrombolysis access for AIS patients. ${ }^{13}$ Saver et al. also found that every 15 -minute increment of a faster onset-to-treatment time was associated with reduced in-hospital mortality, reduced $\mathrm{SICH}$, increased independent ambulation at discharge, and increased discharge to home. ${ }^{14}$ Unfortunately, although the Taiwan HECAL-Stroke project was effective for increasing the in-time treatment rate, the overall $\mathrm{SICH}$ rate was $8.6 \%$, which was higher than the rates of $6.7 \%$ reported in the NINDS rtPA trial $\left.\right|^{4}$ and $7.7 \%$ in a meta-analysis of rtPA treatment in AIS patients. ${ }^{15}$ Previous reports from Albers et al. indicated that the $\mathrm{SICH}$ rate was 3.3\% when $32.6 \%$ of the treated patients violated treatment protocols, $^{16}$ and Katzan et al. found that the SICH rate increased to $15.7 \%$ when $50 \%$ of the treated patients violated the rtPA treatment protocol criteria. ${ }^{17}$ The higher $\mathrm{SICH}$ rate in the study may be attributed to poorer protocol adherence because the overall protocol adherence rate was $87.1 \%$. Additionally, the $\mathrm{MeD}$ group had the lowest protocol adherence rate but the highest $\mathrm{SICH}$ rate compared to the HD and MoD groups. For the limited data reported to MOHW only with an item of adherence or not rather than detailed information, future study is worthy to explore the factors associated with $\mathrm{SICH}$ rate to find out these interesting clinical issues.

Through the efforts of Taiwan's HECAL-Stroke accreditation, the treatment rate increased from $3.0 \%$ to $4.5 \%$ and the in- 
time treatment rate increased from 26\% to 60.1\% from 2009 to 2014. However, the American Heart Association's Get With the Guidelines-Stroke study demonstrated that rtPA treatment less than 3 hours after stroke onset increased from 4.0\% to $7.0 \%$ for all AIS patients and from $42.6 \%$ to $77.0 \%$ in AIS patients arriving at the hospital $\leq 2$ hours after stroke onset from 2003 to $2011 .{ }^{6}$ The HECAL-Stroke project clearly shows that there is still room for improvement in rtPA therapy among Taiwan's AIS patients. According to the stroke chain of survival recommended by the 2013 American Stroke Guidelines, ${ }^{18}$ shortening onset to ER arrival and door to needle time intervals are helpful to improve the rate of thrombolytic therapy for AIS. A previous study in Taiwan also indicated that the time interval between symptom onset and the decision to call for medical care was far from optimal and was the underlying cause of prolonged prehospital delay. ${ }^{19}$ Another study demonstrated that a new designed program with video-assisted therapeutic risk communication significantly decreased the door-to-needle time and increased the percentage of rtPA thrombolytic therapy in patients with AIS. ${ }^{20}$ However, the study did not include the data of onset to ER arrival and door to needle time intervals. Recently, several strategies have been proposed by the MOHW of Taiwan to continually improve access to IV rtPA and to increase the proportion of patients receiving the treatment. At the beginning of 2016, the MOHW of Taiwan began to pay for rtPA treatment to increase financial incentives for hospitals aiming to improve their rtPA administration rates. Additionally, several strategies for the implementation of stroke treatment have been undertaken by the MOHW of Taiwan, such as adding a new indicators "the rate of door to needle time less than 60 minutes" as outcome indicator in HECAL-Stroke project, streamlining pre-hospital stroke management, setting up a telemedicine consulting network for acute stroke management, and processing the approval of mechanical thrombectomy by Taiwan Food and Drug Administration for acute stroke patients with large artery occlusion.

The strength of this study is that this was a nationwide project initiated by the government first for the setting of PSC in Taiwan. However, several limitations should be considered when interpreting the study results. First, the outcome measures were related to rtPA treatment, which might have been affected by the denominator and eligibility criteria when the calculations were performed to detect significant differences. Second, the influence of other stroke public awareness campaigns might have contributed to the improvement of stroke therapy during the study period. Third, the variability in factors explored across different levels of hospital care, such as the interaction between the cause of protocol violation for rtPA treatment and the severity of $\mathrm{SICH}$, was not considered and might have introduced some bias into the study. The development of a broad and detailed framework that can be applied to future studies in this area may be useful.

\section{Conclusions}

The "HECAL-Stroke" project from the MOHW of Taiwan significantly improved rtPA treatment in AIS patients in Taiwan. These findings indicate that a thoughtfully designed and wellreported project initiated by the government to improve stroke treatment quality can be effective in both academic medical centers and community hospital settings. However, the implementation of stroke healthcare not only includes rtPA treatment but also lifestyle modifications, risk factor management, ${ }^{21,22}$ secondary stroke prevention and post-stroke care, and the development of systems such as telestroke and mechanical thrombectomy. Therefore, the integration of stroke services in the future will remain a great challenge for the MOHW of Taiwan.

\section{Supplementary Materials}

Supplementary materials related to this article can be found online at https://doi.org/10.5853/jos.2016.01655.

\section{References}

1. Cheng TJ, Ke DS, Guo HR. The association between arsenic exposure from drinking water and cerebrovascular disease mortality in Taiwan. Water Res 2010;44:5770-5776.

2. Department of Statistics, Ministry of Health and Welfare. Cause of death statistics. http://www.mohw.gov.tw/CHT/ DOS/Statistic.aspx?f_list_no=474\&fod_list_no=3443. 2016. Accessed June 18, 2016.

3. Bluhmki E, Chamorro A, Dávalos A, Machnig T, Sauce $C$, Wahlgren $N$, et al. Stroke treatment with alteplase given 3.0$4.5 \mathrm{~h}$ after onset of acute ischaemic stroke (ECASS III): additional outcomes and subgroup analysis of a randomised controlled trial. Lancet Neurol 2009;8:1095-1102.

4. National Institute of Neurological Disorders and Stroke rt-PA Stroke Study Group. Tissue plasminogen activator for acute ischemic stroke. N Eng/ J Med 1995;333:1581-1587.

5. Hsieh Fl, Lien LM, Chen ST, Bai CH, Sun MC, Tseng HP, et al. Get with the guidelines-stroke performance indicators: surveillance of stroke care in the Taiwan stroke registry: get with the guidelines-stroke in Taiwan. Circulation 2010;122:11161123. 
6. Schwamm LH, Fonarow GC, Reeves MJ, Pan W, Frankel MR, Smith $\mathrm{EE}_{\text {, et }}$ al. Get with the guidelines-stroke is associated with sustained improvement in care for patients hospitalized with acute stroke or transient ischemic attack. Circulation 2009;119:107-115.

7. Lattimore SU, Chalela J, Davis L, DeGraba T, Ezzeddine M, Haymore J, et al. Impact of establishing a primary stroke center at a community hospital on the use of thrombolytic therapy: the NINDS Suburban Hospital Stroke Center experience. Stroke 2003;34:e55-e57.

8. Department of Medical Affairs, Ministry of Health and Welfare. Hospital emergent capability accreditation by level. Ministry of Heal and Welfare. http://www.mohw.gov.tw/CHT/ DOMA/DM1.aspx?f_list_no=935\&fod_list_no=5771. 2016. Accessed May 17, 2016.

9. Alberts MJ, Latchaw RE, Jagoda A, Wechsler LR, Crocco $T$, George $M G$, et al. Revised and updated recommendations for the establishment of primary stroke centers: a summary statement from the brain attack coalition. Stroke 2011;42:2651-2665.

10. Meretoja $A$, Roine RO, Kaste $M$, Linna $M$, Roine $S$, Juntunen $M$, et al. Effectiveness of primary and comprehensive stroke centers: PERFECT stroke: a nationwide observational study from Finland. Stroke 2010;41:1102-1107.

11. Kleindorfer D, Lindsell CJ, Brass L, Koroshetz W, Broderick JP. National US estimates of recombinant tissue plasminogen activator use: ICD-9 codes substantially underestimate. Stroke 2008;39:924-928.

12. Kleindorfer D, de los Rios La Rosa F, Khatri P, Kissela B, Mackey J, et al. Temporal trends in acute stroke management. Stroke 2013;44(6 Suppl 1):S129-S131.

13. Lindsberg PJ, Häppölä 0 , Kallela M, Valanne L, Kuisma $M$, Kaste M. Door to thrombolysis: ER reorganization and reduced delays to acute stroke treatment. Neurology 2006;67:334-336.
14. Saver JL, Fonarow GC, Smith EE, Reeves MJ, Grau-Sepulveda MV, Pan $W$, et al. Time to treatment with intravenous tissue plasminogen activator and outcome from acute ischemic stroke. JAMA 2013;309:2480-2488.

15. Wardlaw JM, Murray V, Berge $E_{1}$ del Zoppo G, Sandercock $P$, Lindley $\mathrm{RL}$, et al. Recombinant tissue plasminogen activator for acute ischaemic stroke: an updated systematic review and meta-analysis. Lancet 2012;379:2364-2372.

16. Albers GW, Bates VE, Clark WM, Bell $R$, Verro $P$, Hamilton $S A$. Intravenous tissue-type plasminogen activator for treatment of acute stroke: the Standard Treatment with Alteplase to Reverse Stroke (STARS) study. JAMA 2000;283:1145-1150.

17. Katzan IL, Furlan AJ, Lloyd LE, Frank Jl, Harper DL, Hinchey $J A$, et al. Use of tissue-type plasminogen activator for acute ischemic stroke: the Cleveland area experience. JAMA 2000;283:1151-1158.

18. Jauch EC, Saver JL, Adams HP, Bruno A, Connors JJ, Demaerschalk BM, et al. Guidelines for the early management of patients with acute ischemic stroke: a guideline for healthcare professionals from the American Heart Association/American Stroke Association. Stroke 2013;44:870-947.

19. Chang KC, Tseng MC, Tan TY. Prehospital delay after acute stroke in Kaohsiung, Taiwan. Stroke 2004;35:700-704.

20. Hsieh CY, Chen WF, Chen $\mathrm{CH}$, Wang $\mathrm{CY}$, Chen $\mathrm{CJ}$, Lai EC, et al. Efforts to reduce the door-to-needle time of thrombolysis in acute ischemic stroke: video-assisted therapeutic risk communication. J Formos Med Assoc 2014;113:929-933.

21. Sung YF, Lee JT, Tsai CL, Lin CC, Hsu YD, Lin JC, et al. Risk factor stratification for intracranial stenosis in Taiwanese patients with cervicocerebral stenosis. J Am Heart Assoc 2015;4:e002692.

22. Sung YF, Lu CC, Lee JT, Hung YJ, Hu CJ, Jeng JS, et al. Homozygous $\mathrm{ALDH}^{*} 2$ is an independent risk factor for ischemic stroke in Taiwanese men. Stroke 2016;47:2174-2179. 
Supplementary Methods 1. Criteria for IV rtPA therapy by National Health Insurance in Taiwan.

Inclusion criteria:

1. Age between 18 and 80 years old

2. Non-contrast computed tomography (CT) scan showing no hemorrhage

3. Acute ischemic stroke with symptoms onset less than 3 hours and complete evaluation

Exclusion criteria:

1. Acute ischemic stroke with symptoms onset more than 3 hours or unknown

2. Rapid improvement of stroke symptoms or stroke severity too mild (the National Institutes of Health Stroke Scale [NIHSS] less than 6)

3. Stroke severity too severe (NIHSS greater than 25 ) or hypodensity greater than $1 / 3$ cerebral hemisphere on CT findings

4. Seizure at onset

5. Recent head trauma, or stroke (less than 3 months)

6. History of stroke with Diabetes mellitus

7. Heparin used in 48 hours before stroke and prolonged aPTT

8. Platelets less than $100,000 / \mathrm{mm} 3$

9. Active internal bleeding

10. Intracranial brain tumor or brain aneurysm or vascular malformation

11. Systolic blood pressure greater than 185 or diastolic blood pressure greater than $110 \mathrm{~mm} \mathrm{Hg}$ or needed to be aggressively treated by IV medication to reach these target levels

12. Glucose less than 50 or greater than $400 \mathrm{mg} / \mathrm{dL}$

13. Patients currently receiving oral anticoagulant, such as Warfarin sodium with PT INR $>1.3$

14. History of intracranial hemorrhage or brain aneurysm or vascular malformation or brain tumor, intracranial or spinal surgery

15. History, suspicion or approval of intracranial hemorrhage, subarachnoid hemorrhage

16. Serious and uncontrolled hypertension

17. Recent surgery, serious trauma or head injury (less than 10 days) including acute myocardia infarct

18. Prolonged or traumatic cardiopulmonary cerebral resuscitation (more than 2 minutes), delivery, recent (less than 10 days) uncompressible vascular puncture (such as subclavian or neck central venous puncture)

19. Severe hepatic diseases, including hepatic failure, liver cirrhosis, portal hypertension (esophageal varicose vein), and acute hepatitis

20. Hemorrhagic retinopathy (such as diabetic), or other hemorrhagic ophthalmic conditions

21. Subacute bacterial endocarditis, acute pericarditis

22. Acute pancreatitis

23. Peptic ulcer disease in recent 3 months

24. Aneurysm, arteriovenous malformation

25. Tumor with easy bleeding

26. Allergy to rt-PA or adjuvant agent

27. Other conditions with increased risk of bleeding, such as hemodialysis, heart failure, cachexia 


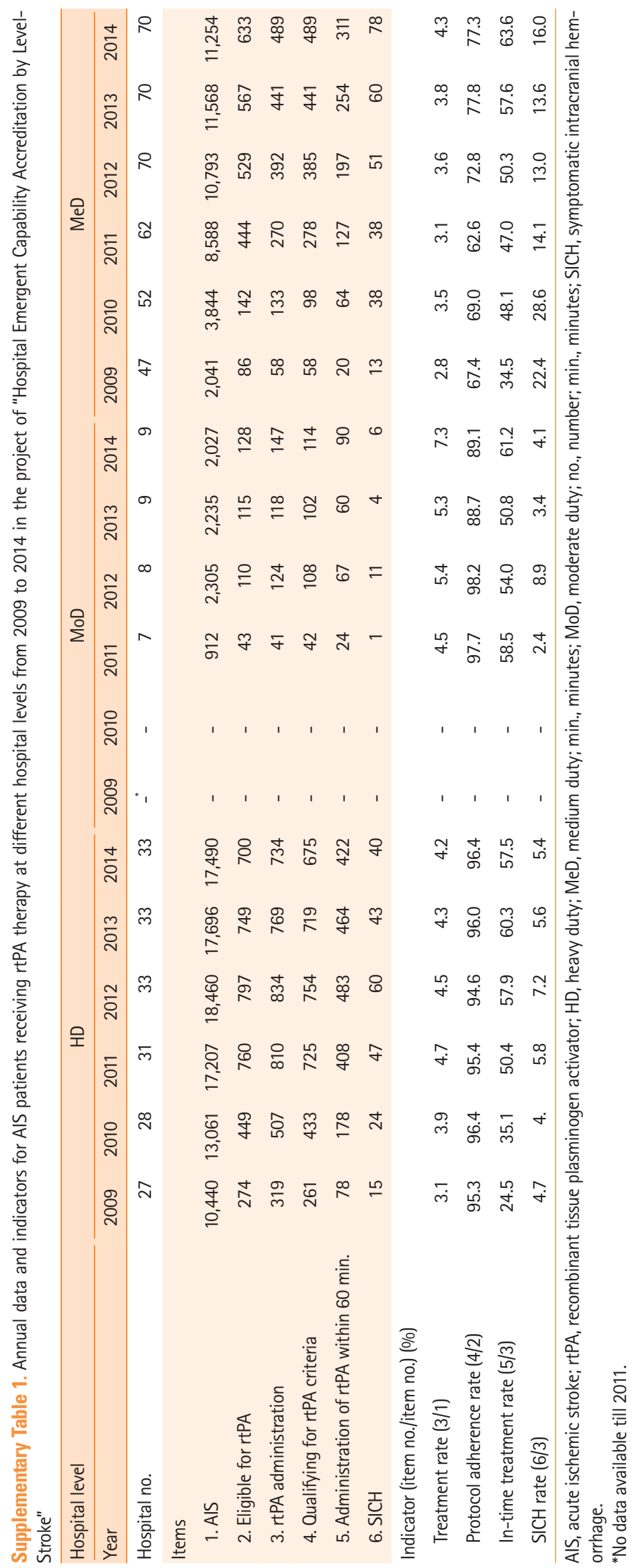




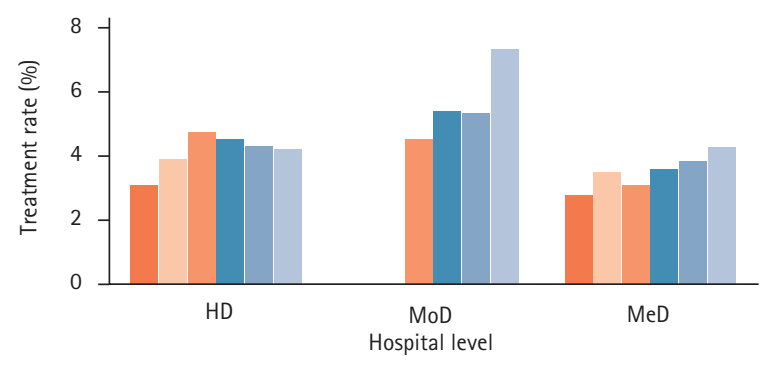

$2009 \square 2010 \square 2011 \square 2012 \square 2013 \square 2014$ (year)

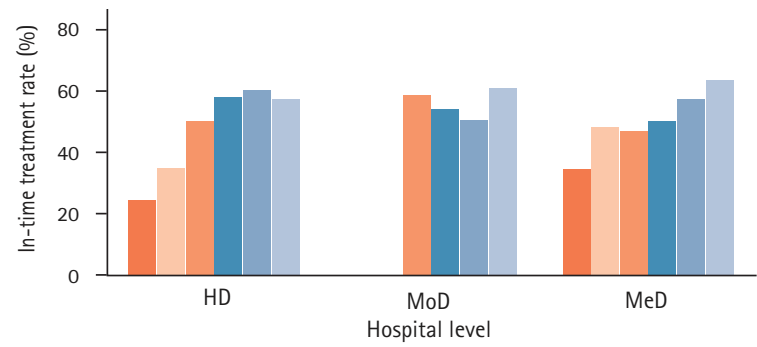

$2009 \square 2010 \square 2011 \square 2012 \square 2013 \quad 2014$ (year)

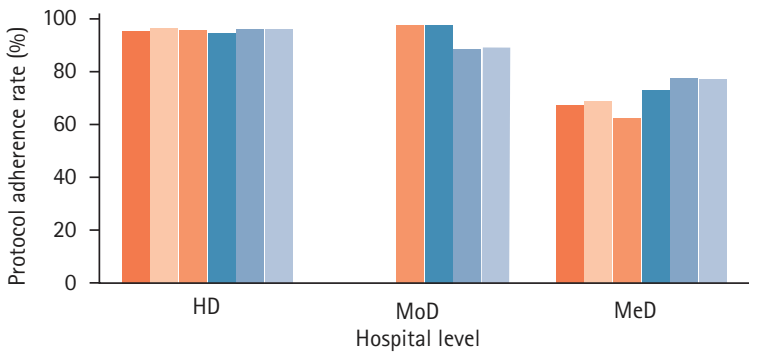

A

$2009 \square 2010 \square 2011 \square 2012 \square 2013 \square 2014$ (year)

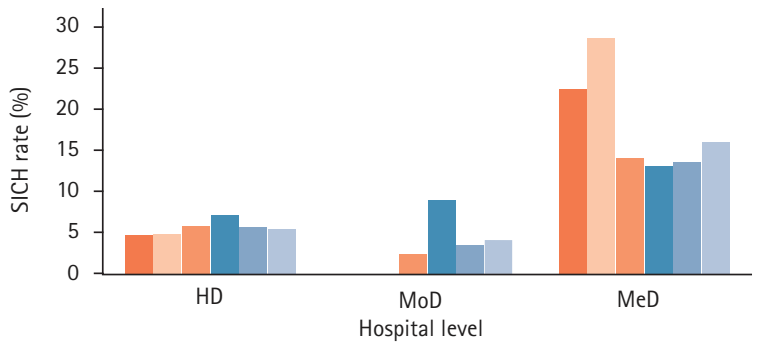

$2009 \square 2010 \square 2011 \square 2012 \square 2013 \square 2014$ (year)
B

(D)

Supplementary Figure 1. Annual indices for AIS patients receiving rtPA therapy at different hospital levels from 2009 to 2014 in the project "Hospital Emergent Capability Accreditation by Level-Stroke". (A)Treatment rate, (B) protocol adherence rate, (C) in-time treatment rate, and (D) SICH rate. AIS, acute ischemic stroke; rtPA, recombinant tissue plasminogen activator; HD, heavy duty; MoD, moderate duty; MeD medium duty; SICH, symptomatic intracranial hemorrhage. 